\title{
Stark broadening of hydrogen lines in low-density magnetized plasmas
}

\author{
J. Rosato* \\ IEF-Plasmaphysik, TEC Euratom Association, Forschungszentrum Jülich GmbH, D-52425 Jülich, Germany \\ Y. Marandet, H. Capes, S. Ferri, C. Mossé, L. Godbert-Mouret, M. Koubiti, and R. Stamm \\ Laboratoire PIIM, UMR 6633, Université de Provence/CNRS, Centre de St-Jérôme, Case 232, F-13397 Marseille Cedex 20, France
}

(Received 22 October 2008; published 27 April 2009)

\begin{abstract}
Stark broadening of hydrogen lines in the presence of a magnetic field is revisited, with emphasis on the role of the ion component under typical conditions of magnetized fusion devices. An impact theory for ions valid at low density $\left(N_{e} \lesssim 10^{14} \mathrm{~cm}^{-3}\right)$ and taking into account the Zeeman degeneracy removal of the atomic states is developed. It is shown that the Stark widths of the Lorentz triplet components strongly depend on the magnetic field. The model is validated by a computer simulation method. For the lateral $\sigma$ components of $\operatorname{Ly} \alpha$, we show that the impact approximation still holds for densities as high as $N_{e} \sim 10^{15} \mathrm{~cm}^{-3}$. In contrast, for the central $\pi$ component as well as for the other lines from low principal quantum number, significant discrepancies between the proposed theory and the simulation results appear at high density. Application to $D \alpha$ in tokamak divertor plasma conditions shows that, in this case, the quasistatic approximation becomes more relevant.
\end{abstract}

DOI: 10.1103/PhysRevE.79.046408

PACS number(s): 52.55.- s, 32.60.+i, 32.70.Jz

\section{INTRODUCTION}

Current spectroscopic investigations of magnetic fusion plasmas have prompted a renewal of interest in spectral lineshape modeling. In the divertor region of the Alcator C-Mod tokamak, the plasma density is sufficiently high $\left(N_{e}\right.$ up to a few $10^{15} \mathrm{~cm}^{-3}$ ) and the temperature is sufficiently low (down to $T_{e}<1 \mathrm{eV}[1]$ ), so that the hydrogen isotope lines routinely measured for diagnostic purposes are affected by Stark broadening. This is the case for both high- $n$ lines [2] (like in other tokamaks, e.g., [3]) and the low- $n$ lines, such as $D \alpha$, for which Doppler, Zeeman, and Stark effects, are of the same order of magnitude [4-6] (here $n$ stands for the principal quantum number of the upper state). Neglecting Stark effect for low- $n$ lines could affect the standard spectroscopic diagnostics foreseen for ITER (International Thermonuclear Experimental Reactor). Retaining only Doppler broadening would, for instance, result in a systematic error in the spectroscopic determination of the recycling flux based on the estimation of the molecular contribution from the atomic line-shape analysis (e.g., [7]). The development of Stark profile models has also been motivated by a need for accuracy in numerical simulations of radiation transport [8]. Simulations of divertor plasmas with edge transport codes, such as B2EIRENE [9], have indeed highlighted the influence of low- $n$ Lyman line photon trapping on the ionization-recombination balance, in Alcator C-Mod [10] as well as in ITER [11,12]. A significant shift of this balance toward ionization would have serious consequences for ITER operation. Therefore, accurate and computationally efficient expressions for StarkZeeman line profiles in the atom's rest frame are required for the description of the photon emission and absorption rates. The "standard" model of Stark broadening, where ions are treated in the quasistatic approximation and electrons within

\footnotetext{
*j.rosato@fz-juelich.de; joel.rosato@univ-provence.fr
}

the impact theory, has already been applied for low- $n$ lines in divertor conditions. The model was suitably modified to account for the effect of the magnetic field such as the anisotropy of the ion microfield distribution or the modification of the emitter energy-level structure due to the Zeeman effect $[13,14]$. The quasistatic treatment of the ions is, however, a contentious issue since its applicability criteria are only marginally satisfied. It is generally assumed to be correct for densities higher than $10^{15} \mathrm{~cm}^{-3}[14,15]$ and calculations with the model microfield method (MMM) did show evidence of ion dynamics effects, on both Lyman and Balmer lines at $10^{15} \mathrm{~cm}^{-3}[16,17]$. In fact, for low- $n$ lines in typical divertor plasma conditions, the validity criteria of the impact approximation for ions $\tau_{c} / \tau_{i}, \rho_{W}^{T} / r_{0} \ll 1$ (with $\tau_{i}, \tau_{c}, r_{0}$, and $\rho_{W}^{T}$, respectively, denoting the time of interest of the line at half maximum, the ion collision time, the mean interparticle distance, and the thermal ion Weisskopf radius) are not very far from being satisfied, so that its use is tempting. However, before doing so two questions remain to be addressed. First, the conventional impact theory for hydrogen lines (see, e.g., [18]), which relies on the calculation of the collision operator made by Griem et al. ([19], Griem, Kolb, and Shen, GKS hereafter), does not retain the energy-level splitting arising from the Zeeman effect. The latter partial degeneracy removal changes the nature of the Stark perturbation, since a part of the Zeeman sublevels is affected by quadratic instead of the linear Stark effect. It can be very significant for ions which here govern the Stark broadening. An approach taking this effect into account has already been developed [20], but an explicit line-shape calculation has not yet been presented for each line components. The second question to be addressed is the accuracy of the impact approximation at the boundary of its validity domain and beyond. When the validity conditions for the impact theory are not fully verified, numerical simulation techniques are suitable [21,22]. This approach has recently been applied to magnetized hydrogen plasmas in order to calculate high- $n$ line shapes, i.e., lines for which the Zeeman effect is negligible in comparison to the 
Stark perturbation [23], but no numerical simulations have been published to date for low- $n$ lines.

In this work, we present calculations for Stark broadening of hydrogen lines for a wide range of tokamak divertor plasma conditions $\left(10^{13} \mathrm{~cm}^{-3} \lesssim N_{e} \lesssim 10^{15} \mathrm{~cm}^{-3}\right)$. We first develop a theory for the collision operator of hydrogen atoms affected by Zeeman effect, using the formalism developed for the nondegenerate levels of the helium atom [24-26] (model of Griem, Baranger, Kolb, and Oertel, GBKO in the following). The consequences of the Zeeman degeneracy removal are discussed on the Lyman $\alpha$ line shape in the atom's rest frame. Next, we present a recently developed numerical simulation code suited for line-shape calculations in weakly coupled magnetized plasmas. We discuss the validity of the impact theory and investigate the intermediate regime, i.e., when neither the impact theory nor the quasistatic approximation should hold, by simulating the profile of $\operatorname{Ly} \alpha$ and $D \alpha$. Consequences for opacity calculations and spectroscopic diagnostics are finally discussed.

\section{STARK IMPACT APPROXIMATION WITH ZEEMAN EFFECT}

\section{A. General formalism}

According to the formalism presented in classical textbooks or review articles (e.g., $[18,27,28]$ ), we start from the formal expression of a line shape $I(\Delta \omega)$ in the atom's rest frame at a frequency detuning $\Delta \omega$, namely,

$$
\begin{gathered}
I(\Delta \omega)=\frac{1}{\pi} \operatorname{Re} \int_{0}^{\infty} C(t) e^{i \Delta \omega t} d t, \\
C(t)=\left\{\operatorname{Tr}\left(\rho_{n} \vec{d}_{\perp} \cdot U_{n^{\prime}}^{\dagger}(t) \vec{d}_{\perp} U_{n}(t)\right)\right\} .
\end{gathered}
$$

Here, $C(t)$ is the dipole autocorrelation function which involves a statistical average $\{\ldots\}$ over the perturber states and a trace $\operatorname{Tr}(\ldots)$ over the atomic states. $\rho_{n}$ is the density operator related to the initial states, $\vec{d}_{\perp}$ is the dipole operator projected into the plane orthogonal to the wave vector $\vec{k}$, and $U_{n}(t)$ [respectively, $\left.U_{n^{\prime}}(t)\right]$ is the evolution operator in interaction representation projected into the subspace related to the initial (respectively, final) states. For hydrogen lines, $n$ and $n^{\prime}$ stand for the principal quantum number of the initial and final states. The evolution operator obeys the Schrödinger equation in interaction representation

$$
\begin{gathered}
i \hbar \frac{d U_{n}}{d t}(t)=-\vec{d}_{n}(t) \cdot \vec{E}(t) U_{n}(t), \\
\vec{d}_{n}(t)=e^{i\left(H_{0}-\vec{\mu}_{n} \cdot \vec{B}\right) t / \hbar} \vec{d}_{n} e^{-i\left(H_{0}-\vec{\mu}_{n} \cdot \vec{B}\right) t / \hbar},
\end{gathered}
$$

where $\vec{\mu}_{n}$ and $\vec{d}_{n}$, respectively, stand for projections of the magnetic moment and the dipole into the subspace with principal quantum number $n . \vec{B}$ is the magnetic field, $\vec{E}(t)$ is the microscopic electric field produced both by the ions and the electrons, and $H_{0}$ is the Hamiltonian of the unperturbed atom. Here, we consider the impact approximation for ions and electrons, i.e., we describe the Stark perturbation such as the result of a series of binary and brief collisions. This leads to replace the Stark effect term $-\vec{d}_{n}(t) \cdot \vec{E}(t)$ by $i \hbar \Phi_{n}$, where $\Phi_{n}$ is the collision operator corresponding to the ion perturbation [at equal temperatures, its electron counterpart is weaker by a factor $\left(m_{e}^{r} / m_{i}^{r}\right)^{1 / 2}, m_{e}^{r}$ and $m_{i}^{r}$ being, respectively, the atom-electron and atom-ion reduced masses]. The collision operator is defined by the following expression [18]:

$$
\Phi_{n}=-N \int d v f(v) v \int d \rho 2 \pi \rho\left\langle 1-S_{n}\right\rangle .
$$

The double integral is performed over the relative velocity $v$ and the impact parameter $\rho$ of the collision, the brackets $\langle\ldots\rangle$ stand for angular average, $N$ and $f(v)$ are, respectively, the plasma density and the distribution function of the ion relative velocities, and $S_{n}$ denotes the scattering matrix related to the evolution of the atom under one binary and complete collision. The collision operator is formally given by expanding $S_{n}$ in a Dyson series, in interaction representation

$$
\begin{aligned}
S_{n}= & 1+\frac{1}{i \hbar} \int_{-\infty}^{+\infty} d t V_{n}(t)+\frac{1}{(i \hbar)^{2}} \int_{-\infty}^{+\infty} d t V_{n}(t) \int_{-\infty}^{t} d t^{\prime} V_{n}\left(t^{\prime}\right) \\
& +\ldots \\
\equiv & \mathcal{T} \exp \left[\frac{1}{i \hbar} \int_{-\infty}^{+\infty} d t V_{n}(t)\right],
\end{aligned}
$$

where $\mathcal{T}$ is the time-ordering operator and $V_{n}(t)$ $=-\vec{d}_{n}(t) \cdot \vec{E}_{i}(t)$ is the interaction potential related to the Stark perturbation corresponding to one ion only. The electric field $\vec{E}_{i}(t)$ is given by the Coulomb expression (nonrelativistic plasma approximation)

$$
\vec{E}_{i}(t)=-\frac{e \vec{r}(t)}{4 \pi \varepsilon_{0}|\vec{r}(t)|^{3}},
$$

$\vec{r}(t)$ denoting the position of the ion under consideration relative to the atom's location. For conditions of tokamak edge plasmas, the ion Larmor radius is much larger than the Debye length. This allows to neglect the cyclotron motion of the perturbers and, therefore, to assume that the perturbers located at the vicinity of the atom move along straight paths, i.e., that $\vec{r}(t)=\vec{\rho}+\vec{v} t$. In the following, we will investigate the diagonal matrix elements $\left\langle n, \alpha\left|\Phi_{n}\right| n, \alpha\right\rangle \equiv \Phi_{n, \alpha}$ of the collision operator using the GBKO theory [24] (the nondiagonal elements can be calculated in the same way [25]). We will also focus on the strong magnetic field regime (PaschenBack effect), valid in tokamak conditions, i.e., consider that fine structure acts only as a perturbation of the Zeeman pattern.

The usual separation between strong (st) and weak $(w)$ collisions allows a splitting of $\Phi_{n, \alpha}$ into two parts,

$$
\begin{gathered}
\Phi_{n, \alpha}=\Phi_{n, \alpha}^{\mathrm{st}}+\Phi_{n, \alpha}^{w}, \\
\Phi_{n, \alpha}^{\mathrm{st}}=-N \int d v f(v) v \int_{0}^{\rho_{n, \alpha}^{\mathrm{st}}} d \rho 2 \pi \rho,
\end{gathered}
$$




$$
\Phi_{n, \alpha}^{w}=N \int d v f(v) v \int_{\rho_{n, \alpha}^{\mathrm{st}}}^{\rho_{\max }} d \rho 2 \pi \rho\left\langle S_{n, \alpha}^{(2)}\right\rangle,
$$

where $S_{n, \alpha}^{(2)}=\left\langle n, \alpha\left|S_{n}^{(2)}\right| n, \alpha\right\rangle$ is the second-order term of the Dyson series (the first order vanishes after averaging over the angles because of isotropy), the cutoff at $\rho_{\max } \simeq \lambda_{D}$ comes from Debye screening of the Coulomb potential, and $\rho_{n, \alpha}^{\text {st }}$ is the radius of strong collisions defined as

$$
\left|\left\langle n, \alpha\left|\left\langle S_{n}^{(2)}\left(\rho_{n, \alpha}^{\mathrm{st}}\right)\right\rangle\right| n, \alpha\right\rangle\right| \simeq 1 .
$$

(A refinement in the analysis could be the study of the changes in the screening length $\rho_{\max }$ when $\rho_{\text {Larmor }}^{\text {electron }}$ is of the order or smaller than $\lambda_{D}$, see, e.g., [29].) According to Ref. [24], the quantity $\left\langle S_{n, \alpha}^{(2)}\right\rangle$ is given by

$$
\left\langle S_{n, \alpha}^{(2)}\right\rangle=-\frac{2}{3} \sum_{\alpha^{\prime}}\left|\frac{\hbar \vec{r}_{n, \alpha \alpha^{\prime}}}{m_{e} \rho v}\right|^{2}\left[\mathcal{A}\left(\frac{\rho \omega_{n, \alpha \alpha^{\prime}}}{v}\right)+i \mathcal{B}\left(\frac{\rho \omega_{n, \alpha \alpha^{\prime}}}{v}\right)\right],
$$

where $\vec{r}_{n, \alpha \alpha^{\prime}}=\left\langle n, \alpha|\vec{r}| n, \alpha^{\prime}\right\rangle$ stands for the matrix elements of the position operator in atomic units; $\mathcal{A}(z)$ and $\mathcal{B}(z)$ are, respectively, even and odd functions defined in terms of the modified Bessel functions of the second kind and $\hbar \omega_{n, \alpha \alpha^{\prime}}$ is the energy difference between the states $|n, \alpha\rangle$ and $\left|n, \alpha^{\prime}\right\rangle$. In the spherical base, the matrix elements $\vec{r}_{n, \alpha \alpha^{\prime}}$ vanish unless the selection rule $m-m^{\prime}=0, \pm 1$ be satisfied [30], so that $\omega_{n, \alpha \alpha^{\prime}}=0, \pm \omega_{Z}$, where $\omega_{Z}=e B / 2 m_{e}$ is the Larmor frequency. The argument of the two functions $\mathcal{A}$ and $\mathcal{B}$ is therefore either zero or $\rho / \rho_{m}$, where $\rho_{m}=v / \omega_{Z}$ is a length which characterizes the perturbation of the energy levels by the Zeeman effect. One can take advantage of this result and write the sum over $\alpha^{\prime}$ more explicitly in Eq. (12), so as to get a more practical form for $\left\langle S_{n, \alpha}^{(2)}\right\rangle$

$$
\left\langle S_{n, \alpha}^{(2)}\right\rangle=-\left(\frac{\rho_{W, n}}{\rho}\right)^{2}\left[K_{n, \alpha}^{\|}+K_{n, \alpha}^{\perp} \mathcal{A}\left(\frac{\rho}{\rho_{m}}\right)-i K_{n, \alpha}^{\prime \perp} \mathcal{B}\left(\frac{\rho}{\rho_{m}}\right)\right] .
$$

Here, $\rho_{W, n}=(2 / 3)^{1 / 2} \hbar n^{2} / m_{e} v$ is the Weisskopf radius corresponding to the velocity $v$ and the coefficients $K_{n, \alpha}^{\|}, K_{n, \alpha}^{\perp}$, and $K_{n, \alpha}^{\prime \perp}$ are given by

$$
\begin{gathered}
K_{n, \alpha}^{\|}=\left(1 / n^{4}\right) \sum_{\alpha^{\prime}}\left|z_{n, \alpha^{\prime} \alpha}\right|^{2}, \\
K_{n, \alpha}^{\perp}=\left(1 / n^{4}\right) \sum_{\alpha^{\prime}}\left[\left|x_{n, \alpha^{\prime} \alpha}^{+}\right|^{2}+\left|x_{n, \alpha^{\prime} \alpha}^{-}\right|^{2}\right], \\
K_{n, \alpha}^{\prime \perp}=\left(1 / n^{4}\right) \sum_{\alpha^{\prime}}\left[\left|x_{n, \alpha^{\prime} \alpha}^{+}\right|^{2}-\left|x_{n, \alpha^{\prime} \alpha}^{-}\right|^{2}\right],
\end{gathered}
$$

with the convention $\quad z_{n, \alpha^{\prime} \alpha}=\left\langle n, \alpha^{\prime}|z| n, \alpha\right\rangle, \quad x_{n, \alpha^{\prime} \alpha}^{ \pm}$ $=\left\langle n, \alpha^{\prime}|(x \pm i y)| n, \alpha\right\rangle / \sqrt{2}$. Clearly, the magnetic field affects the Stark effect corresponding to the projection of the electric field $\vec{E}_{\perp}$ in the plane perpendicular to $\vec{B}$, when the impact parameter $\rho$ becomes comparable to $\rho_{m}$ or larger. Both the width and the position of the Zeeman components change accordingly. In the following, we derive a general expression

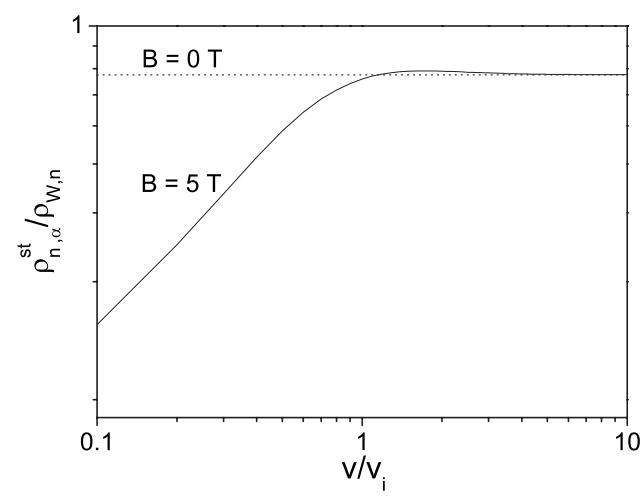

FIG. 1. Plot of the strong collision radius in terms of the velocity, at the temperature $T_{i}=1 \mathrm{eV}$, assuming $B=0$ and $B=5 \mathrm{~T}$ and for $|n, \alpha\rangle=|2, l=1, m=1\rangle$. Discrepancies to the Weisskopf radius are present at low velocities when the magnetic field is finite.

for the collision operator and discuss the physical meaning of the length $\rho_{m}$.

\section{B. Expression of the collision operator}

The collision operator depends on the strong collision radius $\rho_{n, \alpha}^{\text {st }}$ which appears in the integral over the impact parameters [Eqs. (9) and (10)]. When the magnetic field is nonzero, the latter is calculated from Eqs. (11) and (13). For small values of the magnetic field, $\rho_{m}$ remains larger than the Weisskopf radius and the latter gives the strong collision radius up to a numerical factor depending on the line under consideration. However, this is generally not the case in tokamak edge plasma conditions. For deuterium at $v=v_{i}$ (thermal velocity), an estimation shows that $\rho_{m} \sim \rho_{W, n}$ as soon as the ratio $B(\mathrm{~T}) / T_{i}(\mathrm{eV})$ becomes larger than unity. Determining the strong collision radius therefore amounts to solve a transcendental equation, which admits analytical solutions only in the limiting cases where $\rho_{n, \alpha}^{\text {st }} \gg \rho_{m}$ or $\rho_{n, \alpha}^{\text {st }} \ll \rho_{m}$. Figure 1 shows a plot of the ratio $\rho_{n, \alpha}^{\mathrm{st}} / \rho_{W, n}$ in terms of $v / v_{i}$, at $T_{i}=1 \mathrm{eV}$, assuming $B=0$ and $B=5 \mathrm{~T}$ and for $|n, \alpha\rangle=\mid 2, l$ $=1, m=1\rangle$. Whereas the strong collision radius is proportional to $\rho_{W, n}$ in the magnetic field free limit, significant deviations from this simple result do exist at low velocities when the magnetic field is finite. In fact, the ratio $\rho_{n, \alpha}^{\mathrm{st}} / \rho_{W, n}$ follows a power law when $v \ll v_{i}$, as can be demonstrated from the asymptotic behavior of the $\mathcal{A}$ and $\mathcal{B}$ functions.

Once $\rho_{n, \alpha}^{\text {st }}$ is determined, the strong collision term in the collision operator defined in Eq. (9) is calculated from

$$
\Phi_{n, \alpha}^{\mathrm{st}}=-N \int d v f(v) v \pi\left(\rho_{n, \alpha}^{\mathrm{st}}\right)^{2} .
$$

The weak collision term is split into two parts. The first one corresponds to the projection of the electric field on the magnetic field direction $\vec{E}_{\|}=(\vec{E} \cdot \vec{B}) \vec{B} / B^{2}$ and the second one to the projection of the electric field $\vec{E}_{\perp}$ in the plane perpendicular to the magnetic field. The part related to $\vec{E}_{\|}$, which we will denote $\Phi_{n, \alpha}^{w \|}$, is left unaffected by the magnetic field. In fact, performing the integral over the impact parameters yields 


$$
\Phi_{n, \alpha}^{w \|}=-2 K_{n, \alpha}^{\|} N \int d v f(v) v \pi \rho_{W, n}^{2} \ln \left(\frac{\lambda_{D}}{\rho_{n, \alpha}^{\mathrm{st}}}\right) .
$$

The logarithm dependence is the same as obtained in the GKS theory for the hydrogen atom in the magnetic field free case [19]. As a difference with GKS which uses an average cutoff at $\rho_{W, n}$, we retain in our calculation the full quantum number dependence provided by $\rho_{n, \alpha}^{\mathrm{st}}$, an expression already given in their paper [see Eqs. (18) and (19) of Ref. [19]]. Equation (16) shows that the Zeeman effect does not affect the Stark broadening arising from $\vec{E}_{\|}$. Conversely, the part related to $\vec{E}_{\perp}$, which we will denote $\Phi_{n, \alpha}^{w \perp}$, depends on the magnetic field through the length $\rho_{m}$ and does not involve the usual Coulomb logarithm appearing in Eq. (16). The integral over the impact parameter can be performed formally by introducing the functions $a(z)$ and $b(z)$ defined in [24] for the helium atom. Explicitly, one obtains

$$
\begin{aligned}
\Phi_{n, \alpha}^{w \perp}= & -\left.2 K_{n, \alpha}^{\perp} N \int d v f(v) v \pi \rho_{W, n}^{2} a(z)\right|_{\lambda_{D}^{\prime} / \rho_{m}} ^{\rho_{n, \alpha}^{\mathrm{st}} / \rho_{m}} \\
& +\left.2 i K_{n, \alpha}^{\prime \perp} N \int d v f(v) v \pi \rho_{W, n}^{2} b(z)\right|_{\lambda_{D}^{\prime} / \rho_{m}} ^{\left.\right|_{n,} ^{\mathrm{st}} \alpha \rho_{m}} .
\end{aligned}
$$

The functions $a(z)$ and $b(z)$ vanish at large values of their argument $z$, respectively, such as $(\pi / 2) e^{-2|z|}$ and $\pi / 4 z$, and satisfy $a(z) \sim \ln (1 /|z|)$ and $b(z) \rightarrow \pm \pi / 2$ when $z \rightarrow 0^{ \pm}$. The expression (17) thus leads to the usual collision operator for the hydrogen atom when $\rho_{m} \rightarrow+\infty$, i.e., when the magnetic field vanishes. An interesting case is that obtained when $\rho_{W, n} \ll \rho_{m} \ll \lambda_{D}$, namely,

$$
\begin{aligned}
\Phi_{n, \alpha}^{w \perp}= & -2 K_{n, \alpha}^{\perp} N \int d v f(v) v \pi \rho_{W, n}^{2} \ln \left(\frac{\rho_{m}}{\rho_{W}}\right) \\
& +i \pi K_{n, \alpha}^{\prime \perp} N \int d v f(v) v \pi \rho_{W, n}^{2} .
\end{aligned}
$$

Here, a Coulomb logarithm appears in the real part, and the magnetic length $\rho_{m}$ plays the role of a cutoff at large impact parameter. From a classical mechanics point of view, this cutoff is related to the Larmor precession since $\rho_{m}$ is the typical distance covered by a perturber during one precession of the classical angular momentum. Its presence in the logarithm effectively reduces the duration of a collision to the order of the Larmor period. This can be understood as follows. In the plane perpendicular to the magnetic field, the projection of the dipole is rotating at a frequency $\omega_{Z}$ so that the corresponding interaction potential $V(t)$ oscillates at $\omega_{Z}$. If the inverse of this frequency (i.e., the time it takes to perform a Larmor precession) is much shorter than the Debye time $\tau_{D} \sim \lambda_{D} / v=\omega_{p}^{-1}$ [i.e., the time during which $V(t)$ takes a non-negligible value] then the duration of the collision is effectively shortened in the sense that the time integration in Eq. (6) has to be performed on a duration of the order of $\omega_{Z}^{-1}$ instead of $\omega_{p}^{-1}$. In other words, in the frame of the rotating dipole, the electric field vanishes in the average on a time scale of the order of $\omega_{Z}^{-1}$. From a practical point of view, this cutoff reduces the collisional broadening of the Zeeman components and intuitively should extend the valid-

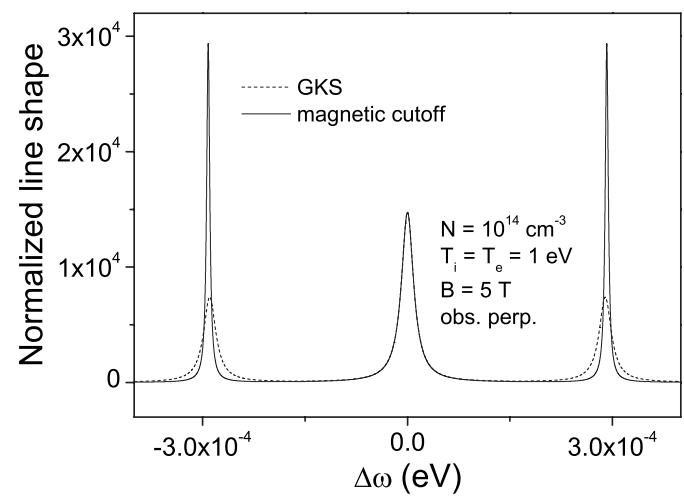

FIG. 2. Plot of the Lyman $\alpha$ line in the atom's rest frame obtained with the impact theory, including the magnetic cutoff (solid line) or not (dashed line). The magnetic cutoff strongly reduces the Stark broadening corresponding to the perpendicular electric field, which controls the width of the lateral components in the case of Lyman $\alpha$.

ity domain of the impact approximation because fast oscillations of the electric field may be treated as collisions. To illustrate this result, we have calculated the profile of Lyman $\alpha$ in the atom's rest frame by using the expression (17) for the contribution of the perpendicular ion electric field. Figure 2 displays the Zeeman triplet obtained in perpendicular observation, assuming $N=10^{14} \mathrm{~cm}^{-3}, T_{e}=T_{i}=1 \mathrm{eV}$, and $B$ $=5 \mathrm{~T}$ (typical conditions expected in the divertor plasma of ITER). The profile obtained using the GKS theory for ions, i.e., calculating the Stark profile in the magnetic field free case and folding with the Zeeman pattern, is also plotted. The broadening of the central component is well described by the GKS formalism even in the presence of a magnetic field (this behavior is unique, as pointed out in [20]). In contrast, the lateral components, which are only broadened by the perpendicular electric field, are much narrower than expected from the GKS theory if $\rho_{m} \ll \lambda_{D}$ (for the conditions under consideration here, we obtain $\rho_{m} / \lambda_{D} \simeq 4 \%$ ).

\section{ASSESSMENT OF THE INTERMEDIATE REGIME}

The impact approximation no longer holds as soon as at least one of the strong inequalities $\tau_{i} \gg \tau_{c}$ or $r_{0} \gg \rho_{W}^{T}$ is not fully verified. In this case, either incomplete collision or many-body effects start to play a role. The theoretical approaches devised to tackle the problem of incomplete collisions (Lewis' cutoff [31]; unified theory [32,33]) are able to reproduce the transition to the static limit in the line wings (where the time of interest becomes very short) but do not take many-body effects into account. We have written a computer simulation routine accounting for ion dynamics in the presence of Zeeman effect [34], following the same method as that previously developed in [21]. In this routine, the electric field is simulated according to a quasiparticle model with a Debye screened potential (in accordance with the assumption of a neutral emitter and with the ordering $\lambda_{D} \ll \rho_{\text {Larmor }}^{\text {ion }}$ ). The simulation is performed in a cubic cell with periodic boundary conditions. For each history of the electric field, the routine solves the time-dependent Schrödinger equation 
for the evolution operator $U_{n}(t)$ according to the following algorithm:

$$
U_{n}(t+\Delta t)=U_{n}(t+\Delta t, t) U_{n}(t),
$$

with $U_{n}(t+\Delta t, t)$ being the infinitesimal evolution operator. The same algorithm is used for the determination of $U_{n^{\prime}}(t)$. We have tested various techniques (namely, a Euler method with variable time steps, a Runge-Kutta method [35], and a matrix exponential evaluated by the scaling squaring method [36]) whose solutions agree well between each other. The statistical average required for the line-shape calculation is formed after the determination of $U_{n}(t)$ and $U_{n^{\prime}}(t)$ for various microfield realizations corresponding to independentparticle simulations.

The impact limit is attained as soon as the criteria $\tau_{i}$ $\gg \tau_{c}$ and $r_{0} \gg \rho_{W}^{T}$ are satisfied. In this case, one has $U_{n}(t$ $+\Delta t, t) \simeq S_{n}$ and a relation similar to Eq. (19), involving the collision operator $\Phi_{n}$, holds for the average evolution operator $\left\{U_{n}(t)\right\}$ at a time scale $\Delta t$ larger than the collision time [18]

$$
\left\{U_{n}(t+\Delta t)\right\}=\left(1+\Phi_{n} \Delta t\right)\left\{U_{n}(t)\right\} .
$$

This is precisely the discrete form of the differential equation satisfied by the evolution operator in the impact approximation. We have tested the magnetic cutoff impact (MCI) theory developed in Sec. II by performing simulations at low density, when the validity conditions for impact are satisfied. The two approaches have been found to be in excellent agreement. This is shown in Fig. 3, which displays (a) the red lateral and (b) the central component of Lyman $\alpha$ simulated with $N=10^{13} \mathrm{~cm}^{-3}, T_{e}=T_{i}=1 \mathrm{eV}, B=5 \mathrm{~T}$ (i.e., conditions for which $\rho_{W}^{T} / r_{0} \simeq 6 \%$ and $\tau_{c} / \tau_{i} \simeq 1 \%$ ), as well as the result of the MCI theory and GKS. The simulation was performed with 1000 different statistical realizations for the electric field. As can be seen in Fig. 3(a), GKS strongly overestimates the width of the lateral component, while the narrower profile obtained retaining the magnetic cutoff agrees nicely with the simulation result. This result validates the narrowing expected by the MCI theory and presented in Sec. II B. Conversely, the central component plotted in Fig. 3(b) is still very well described by the GKS theory, again a result expected for Ly $\alpha$ as discussed in Sec. II B. This comparison provides a cross-check between our analytical and numerical approaches for conditions for which the impact theory is known to hold.

At higher densities, the validity conditions of the impact approximation are no longer satisfied, so that nonbinary and incomplete collisions have to be taken into account. To quantify the largest deviations to be expected in divertor conditions for opacity study applications [11,12], we performed a simulation of Lyman $\alpha$ at a plasma density $N=10^{15} \mathrm{~cm}^{-3}$ and with the same values for the temperatures and the magnetic field as above, namely, $T_{e}=T_{i}=1 \mathrm{eV}$ and $B=5 \mathrm{~T}$. According to these values, the impact approximation should only marginally hold since one has $\rho_{W}^{T} / r_{0} \simeq 0.3$. Figure 4 shows a comparison between the MCI theory and the simulation results. The central Zeeman component is clearly narrower than what is obtained using the impact approximation. However, it should be noted that the lateral components ob-
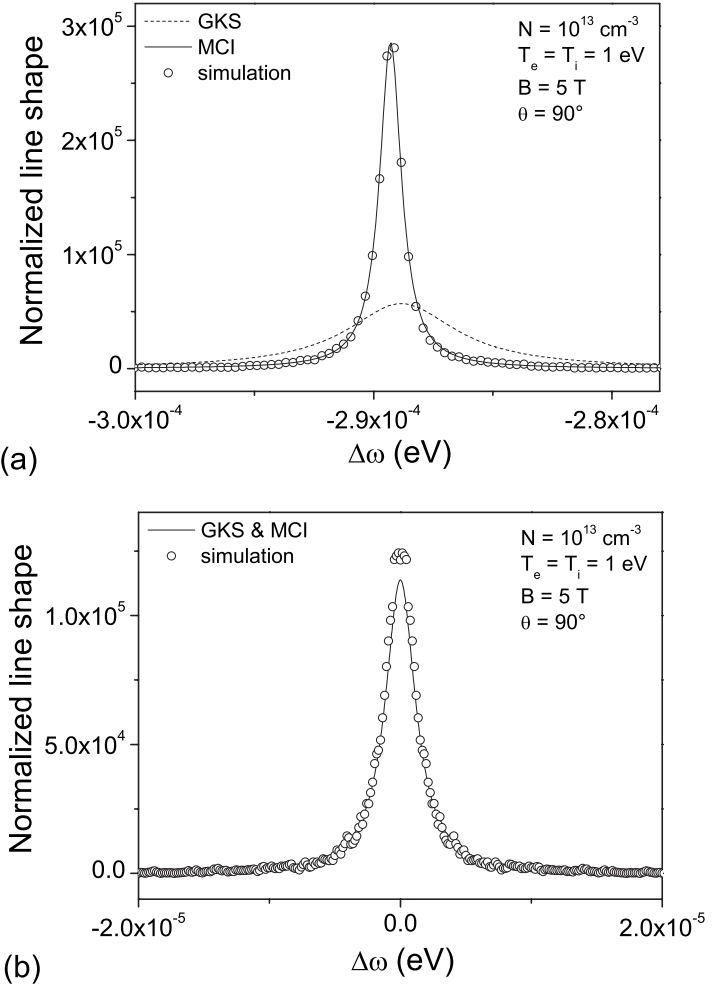

FIG. 3. Plot of (a) the red Zeeman lateral component and (b) the central component of Lyman $\alpha$, in conditions where the impact validity conditions are fully verified. The impact theory with magnetic cutoff (MCI, solid line) is in good agreement with the simulation, whereas the conventional theory for hydrogen (GKS, dashed line) strongly overestimates the linewidth. In the central component case, the perpendicular electric field does not perturb the emitting energy sublevel so that MCI reduces to GKS.

tained by the MCI theory remain in a good agreement with the simulation. This is a consequence of the magnetic cutoff already mentioned above. In fact, the time of interest related to these components is larger than the one obtained from the

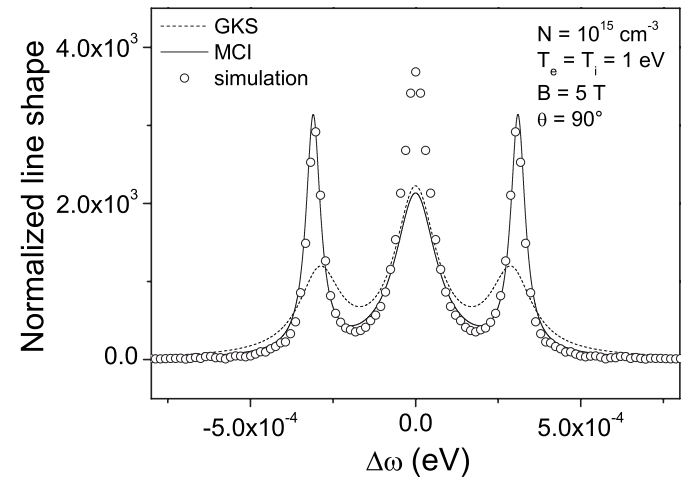

FIG. 4. Lyman $\alpha$ Zeeman-Stark profile obtained with the simulation routine (circles) compared with the result of the MCI theory (solid line), in conditions where the validity conditions of the impact theory are not fully verified. The MCI theory predicts a central component with full width at half maximum larger than that expected from the simulation, but the lateral components remain in a good agreement. 


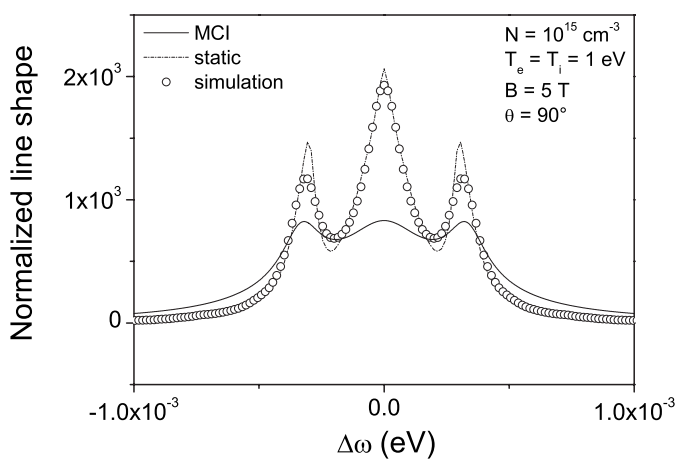

FIG. 5. Plot of $D \alpha$ obtained by the simulation method (circles) compared to the MCI theory (solid line) and to the quasistatic approximation (dash-dotted line). The impact theory strongly overestimates the Stark broadening outside of its validity domain and leads to a loss of structure on the line, whereas the quasistatic profile is found to be in a better agreement.

GKS impact calculation because broadening by the perpendicular electric field is less efficient than in the magnetic field free case. The impact approximation for the lateral components thus holds for densities higher than expected from its usual validity criteria.

Nonimpact effects (i.e., nonbinary and/or incomplete collisions) become more and more significant as the principal quantum number increases, so that the deviations to the impact theory should be more pronounced on the Balmer lines which are used for diagnostic purposes. This is precisely the case for $D \alpha$ for conditions of dense divertor plasmas. We have calculated the profile of $D \alpha$ with the simulation routine and compared the result to both the impact and the quasistatic approximations. Figure 5 shows the profile obtained for $N=10^{15} \mathrm{~cm}^{-3}, T_{e}=T_{i}=1 \mathrm{eV}$, and $B=5 \mathrm{~T}$, in an observation perpendicular to $\vec{B}$. In these conditions, one has roughly $\rho_{W}^{T} / r_{0}=0.6$. As shown in the figure, the quasistatic approximation is in this case closer to the simulation result than the impact theory, which strongly overestimates the Stark broadening and leads to a loss of structure of the Lorentz triplet. It should be noted that the quasistatic theory underestimates the width of the Zeeman components (in particular, the lateral ones). This difference can be explained by the additional broadening of the simulated profiles due to ion dynamics. However, such a discrepancy is not so critical in practice for diagnostics relying on passive spectroscopy because of Doppler broadening. This point is illustrated in Fig. 6, where the profile in the laboratory frame $I_{\text {lab }}(\Delta \omega)$ is plotted for the same conditions as above. The latter is obtained by convolving the Zeeman-Stark profile with the velocity distribution function (VDF) of the emitters $f_{0}(\vec{v})$, according to

$$
I_{\mathrm{lab}}(\Delta \omega)=\int d^{3} \vec{v} f_{0}(\vec{v}) I\left(\Delta \omega-\vec{v} \cdot \vec{n} \omega_{0} / c\right),
$$

where $\vec{n}, \omega_{0}$, and $c$, respectively, stand for the photon propagation direction, the central frequency, and the speed of light. We assume that $f_{0}(\vec{v})$ is Maxwellian at the emitter temperature $T_{0}=1 \mathrm{eV}$, i.e., that neutrals are thermalized with the plasma, which is justified for such a high density (note that

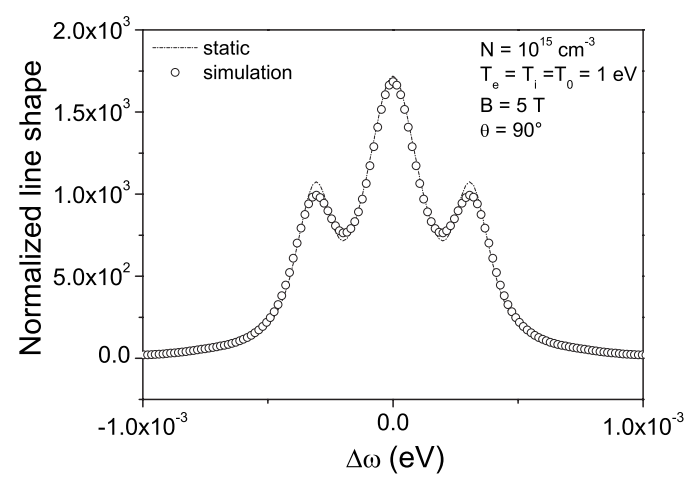

FIG. 6. Plot of $D \alpha$ in the laboratory frame (i.e., including Doppler effect) obtained by the simulation method (circles) compared to the quasistatic approximation. The latter provides a good approximation for the Stark broadening description when the density is high.

for lower densities, a lower temperature of the order of 0.3 $\mathrm{eV}$ corresponding to molecular dissociation processes can even be expected [37]). As shown in Fig. 6, the Doppler effect is strong enough to mask the differences between the two profiles. This result demonstrates that the quasistatic approximation for ions can safely be used for $D \alpha$ spectroscopy in dense divertor conditions. This result was not supported by solid evidence in the literature previously published.

\section{CONCLUSION}

We have investigated Stark profiles of hydrogen lines for low-density magnetized plasmas, focusing on tokamak divertor relevant conditions. At the lowest densities, the validity conditions of the impact theory are satisfied for the ions. This is interesting since the impact theory provides a quasianalytical result, which is handy to use and computationally efficient. We derived a collision operator following the same approach as the one developed for the helium atom. This operator takes into account the degeneracy removal arising from the Zeeman effect. For the hydrogen atom, we have shown that the lateral components of a Lorentz triplet can be strongly narrower than predicted by the impact approximation in the magnetic field free case. The magnitude of this effect depends on how the so-called magnetic length, which acts as a cutoff for impact parameters, compares to the Debye length and the strong collision radius. The narrowing effect has been confirmed by numerical simulations, and the intermediate regime (at higher densities) where the validity criteria of the impact theory are no longer strictly satisfied has been investigated. A numerical simulation code has been developed to include combined Stark and Zeeman effects and compared to those from the impact theory and the quasistatic approximation. The results depend on the line under consideration and differ even between the Zeeman components of the same line. For $\operatorname{Ly} \alpha$, which plays an important role in the opacity of the plasma, the impact approximation remains applicable in the whole range of divertor conditions up to $N_{e}=10^{15} \mathrm{~cm}^{-3}$ for the lateral $\sigma$ components, whereas the central $\pi$ component is already narrower than expected by the impact approximation even at $N_{e}=10^{14} \mathrm{~cm}^{-3}$. This 
difference of behavior is well understood and stems from the fact that smaller linewidth means larger time of interest, hence better applicability of the impact theory for the lateral components. For $D \alpha$, which is used in spectroscopic diagnostics, the quasistatic profile is found to be in a better agreement with the simulation, at least for high densities $\left(10^{15} \mathrm{~cm}^{-3}\right)$. A further step would be to properly retain the motional Stark effect corresponding to the thermal motion of the emitters. In practice, this would mean calculating a Zeeman-Stark profile for each emitter velocity [38]. Any other static electric field leading to an additional Stark effect (as observed in T-tube discharges, e.g., [39]) could be retained in the same way. The numerical simulation method, which provides a reference on line-shape description, cannot be used in a real-time diagnostic since it is time consuming.
Another possible extension of the present work could consist on the establishment of analytical or rapid numerical techniques (e.g., along the line of [40]) able to describe the regime in between the impact and quasistatic limits in the presence of a magnetic field.

\section{ACKNOWLEDGMENTS}

This work was partially supported by the project "Radiation Absorption Effects" of the French Fédération de Recherche sur la Fusion Magnétique, by the project "PHOTONITER" of the French Agence Nationale de la Recherche (Contract No. ANR-07-BLAN-0187-01), and by the collaboration LRC DSM 99-14 (PIIM/CEA Cadarache).
[1] B. Lipschultz, J. L. Terry, C. Boswell, A. Hubbard, B. LaBombard, and D. A. Pappas, Phys. Rev. Lett. 81, 1007 (1998).

[2] B. L. Welch, H. R. Griem, J. Terry, C. Kurz, B. LaBombard, B. Lipschultz, E. Marmar, and G. McCracken, Phys. Plasmas 2, 4246 (1995).

[3] M. Koubiti, S. Loch, H. Capes, L. Godbert-Mouret, Y. Marandet, A. Meigs, R. Stamm, and H. Summers, J. Quant. Spectrosc. Radiat. Transf. 81, 265 (2003).

[4] J. Ghosh, H. R. Griem, R. C. Elton, J. L. Terry, E. Marmar, B. Lipschultz, B. LaBombard, J. E. Rice, and J. L. Weaver, Phys. Plasmas 11, 1033 (2004).

[5] B. L. Welch, J. L. Weaver, H. R. Griem, W. A. Noonan, J. Terry, B. Lipschultz, and C. S. Pitcher, Phys. Plasmas 8, 1253 (2001).

[6] J. L. Weaver, B. L. Welch, H. R. Griem, J. Terry, B. Lipschultz, C. S. Pitcher, S. Wolfe, D. A. Pappas, and C. Boswell, Rev. Sci. Instrum. 71, 1664 (2000).

[7] A. Escarguel et al., Plasma Phys. Controlled Fusion 43, 1733 (2001).

[8] J. Rosato, R. Stamm, L. Godbert-Mouret, M. Koubiti, Y. Marandet, F. Rosmej, and H. Capes, J. Nucl. Mater. 363-365, 421 (2007).

[9] D. Reiter, M. Baelmans, and P. Börner, Fusion Sci. Technol. 47, 172 (2005).

[10] S. Lisgo et al., J. Nucl. Mater. 337-339, 139 (2005).

[11] V. Kotov, D. Reiter, A. S. Kukushkin, H. D. Pacher, P. Börner, and S. Wiesen, Contrib. Plasma Phys. 46, 635 (2006).

[12] D. Reiter, V. Kotov, P. Börner, K. Sawada, R. K. Janev, and B. Küppers, J. Nucl. Mater. 363-365, 649 (2007).

[13] Nguyen-Hoe, H.-W. Drawin, and L. Herman, J. Quant. Spectrosc. Radiat. Transf. 7, 429 (1967).

[14] M. L. Adams, R. W. Lee, H. A. Scott, H. K. Chung, and L. Klein, Phys. Rev. E 66, 066413 (2002).

[15] G. Mathys, Astron. Astrophys. 139, 196 (1984).

[16] S. Günter and A. Könies, J. Quant. Spectrosc. Radiat. Transf. 62, 425 (1999).

[17] C. Stehlé, S. Brillant, and G. Mathys, Eur. Phys. J. D 11, 491 (2000).

[18] H. R. Griem, Spectral Line Broadening by Plasmas (Academic
Press, New York, 1974).

[19] H. R. Griem, A. C. Kolb, and K. Y. Shen, Phys. Rev. 116, 4 (1959).

[20] A. Derevianko and E. Oks, Phys. Rev. Lett. 73, 2059 (1994).

[21] R. Stamm, E. W. Smith, and B. Talin, Phys. Rev. A 30, 2039 (1984).

[22] M. A. Gigosos, J. Fraile, and F. Torres, Phys. Rev. A 31, 3509 (1985).

[23] E. Stambulchik, S. Alexiou, H. R. Griem, and P. C. Kepple, Phys. Rev. E 75, 016401 (2007).

[24] H. R. Griem, M. Baranger, A. C. Kolb, and G. Oertel, Phys. Rev. 125, 177 (1962).

[25] C. Deutsch, L. Herman, and H.-W. Drawin, Phys. Rev. 178, 261 (1969).

[26] C. Deutsch, Phys. Rev. A 2, 1258 (1970).

[27] M. Baranger, Atomic and Molecular Processes (Academic Press, New York, 1962).

[28] V. S. Lisitsa, Sov. Phys. Usp. 20, 603 (1977).

[29] D. Naujoks, Contrib. Plasma Phys. 41, 375 (2001).

[30] H. A. Bethe and E. E. Salpeter, Quantum Mechanics of Oneand Two-Electron Atoms (Springer-Verlag, Berlin, 1957).

[31] M. Lewis, Phys. Rev. 121, 501 (1961).

[32] D. Voslamber, Z. Naturforsch. 24a, 1458 (1969).

[33] E. W. Smith, J. Cooper, and C. R. Vidal, Phys. Rev. 185, 140 (1969).

[34] J. Rosato, Ph.D. thesis, University of Provence, 2007.

[35] S. E. Koonin, Computational Physics (Addison-Wesley, Reading, MA, 1986).

[36] C. Moler and C. V. Loan, SIAM Rev. 45, 3 (2003).

[37] J. D. Hey, C. C. Chu, and E. Hintz, J. Phys. B 32, 3555 (1999).

[38] S. Brillant, G. Mathys, and C. Stehlé, Astron. Astrophys. 339, 286 (1998).

[39] Z. Mijatović, S. Djurović, M. Pavlov, R. Kobilarov, and B. T. Vujičić, Contrib. Plasma Phys. 35, 453 (1995).

[40] L. Godbert-Mouret, M. Koubiti, R. Stamm, K. Touati, B. Felts, H. Capes, Y. Corre, R. Guirlet, and C. D. Michelis, J. Quant. Spectrosc. Radiat. Transf. 71, 365 (2001). 